\title{
The Effect of Saturated Fat Intake With Risk of Stroke and Coronary Heart Disease: A Systematic Review of Prospective Cohort Studies
}

\author{
Hilal Salim Al Shamsi ${ }^{1}$ \& Abdullah Ghthaith Almutairi ${ }^{2}$ \\ ${ }^{1}$ Directorate of Planning and studies in al Buraimi Governorate, Directorate General of Health Services, Ministry \\ of Health, Oman \\ ${ }^{2}$ Quality and Patient Safety Department, Prinice Nasser Bin Saad Hospital, Al-Ghat Province, Ministry of Health, \\ Saudi Arabia \\ Correspondence: Hilal Salim Al Shamsi, Directorate of Planning and studies in al Buraimi Governorate, \\ Directorate General of Health Services, Ministry of Health, Oman.
}

Received: May 9, 2018 Accepted: July 2, 2018 Online Published: July 11, 2018

doi:10.5539/gjhs.v10n8p28 URL: https://doi.org/10.5539/gjhs.v10n8p28

\begin{abstract}
Background: A decline in saturated fat intake has generally been thought to improve cardiovascular health.

Objective: The objective of this review is to summarize the evidence presented in recent prospective epidemiologic studies related to the association of saturated fat intake and risk of stroke and coronary heart disease (CHD).

Design: Sixteen prospective cohort studies identified by searches of the Medline and ProQuest databases are included in this review. The association of saturated fat intake with stroke and CHD risk is explored using the relative risk (RR), Hazard ratio (HR), and 95\% confidence interval (CI) methods.

Results: Over follow-up periods of 8 to 30 years, 22,773 of 668,082 participants of these 16 studies developed stroke or CHD. Saturated fat intake was associated with an increased risk of CHD (HR = 2.36, 95\% CI 1.10-5.09) but not with stroke. Gender and age had no impact on the stroke rate, whereas the female gender was a risk factor for CHD (HR = 3.07, 95\% CI 1.54-6.11). In addition, a subgroup analysis showed a positive association between smoking history and increasing risk of stroke and CHD.
\end{abstract}

Conclusion: This systematic review of prospective-cohort epidemiologic studies found that there is a weak to strong association between saturated fat intake and increased CHD risk but not significant evidence for concluding that saturated fat intake is associated with an increased risk of stroke. In addition, more research is needed to determine whether risk of stroke and/or CHD is potentially affected by specific nutrients used to replace saturated fat.

Keywords: adult, elderly, cardiovascular disease, mortality, sex differences, fats, risk factors
Abbreviations
CVD Cardiovascular disease
CHD Coronary heart disease
MFUAS Monounsaturated fatty acids
PUFAs Polyunsaturated fatty acids

\section{Introduction}

In the last few decades, noncommunicable diseases have become a global concern, with cardiovascular disease (CVD) rising rapidly to the top of the list. CVD can be defined as any disease of the blood vessels or heart (The Department of Health, 2016) and therefore includes heart failure, heart disease, congenital heart disease, coronary heart disease (CHD) and stroke. The most common symptoms of CVD are discomfort or pain in the centre of the chest, vomiting, difficulty breathing and numbness of the face or arm (World Health Organization [WHO], 2017). There are no symptoms for disease in the blood vessels, so a stroke or heart attack is often the first warning that there is a problem.

CVD is a serious illness that contributes significantly to the rates of sickness and death worldwide. The World 
Health Organization [WHO] (2017) has classified CVD as the primary cause of death globally, as it accounts for $31 \%$ of all deaths. In many ways, CVD can also be considered the most costly disease, with the highest level of related health expenditures. For instance, CVD was the main cause of the 475,000 hospitalisations from 2007 to 2008 in Australia (Australian Institute of Health and Welfare [AIHW], 2011). In addition, from 2004 to 2005, CVD cost about AUD 5.9 billion in Australia alone, with over half of this money being spent on hospital admissions (2011). Furthermore, this disease also imposes a significant burden of the overall quality of life, including high rates of disability and premature death. The World Health Organization [WHO] (2004) has pointed out that in 2004, CVD led to 46.6 million disability-adjusted life years worldwide.

Many risk factors are associated with CVD, such as age, sex, family history and behavioural factors. Ram and Trivedi (2012) have highlighted that the majority of CVD cases are caused by behaviour risk factors which can be treated, controlled and modified, such as physical activity, alcohol use, unhealthy diet and saturated fat intake. This paper focuses on one of the behavioural risk factors for CVDs, namely saturated fat. In recent years, epidemiological studies have found an association between dietary saturated fat and increased CVDs risk (Boniface \& Tefft, 2002). On the other hand, other studies have shown no association of saturated fat intake with CVD-related morbidity and mortality (Boniface \& Tefft, 2002; Tucker et al., 2005). The differences in these studies' findings could be due to the effects of some study participants eating less saturated fat but replacing it various other types of polyunsaturated fat. Therefore, this difference in diet could be a cause of increased or reduced CVD risk.

The most serious and common types of CVD are stroke and CHD in many countries (WHO, 2009). The World Health Organization [WHO] (2017) has estimated that globally, 17.7 million people died due to CVD in 2015, 42\% from CHD and $38 \%$ from stroke. Both stroke and CHD may be caused by the same issue; i.e., blockages in blood vessels preventing blood from flowing to the brain or heart. The most common cause for these diseases is the building-up of fatty material (i.e., saturated fat) on the inner walls of vessels that are response to supply blood to brain or heart. Therefore, the goal of this study is to conduct a systematic review of previous studies to estimate the risk of stroke and CHD and to evaluate the association of this risk with an increase of dietary saturated fat.

\section{Method}

\subsection{Study Selection}

A systematic review was conducted of published articles on topics related to saturated fat intake and CVD, stroke and CHD risk. Two databases were used in this review (Medline and ProQuest) with the following keywords: 'saturated fat', 'cardiovascular', 'stroke', 'coronary', 'follow up' and 'cohort'. The search strategy was to compile a list of all prospective cohort studies published between 2000 and 2017.

\subsection{Inclusion and Exclusion Criteria}

This review only includes the prospective cohort studies in which participants were generally healthy at the study baseline and were followed for at least 8 years. There were no restrictions on the type of dietary assessment method used. Only studies that investigated the possible association between saturated fat intake and risk of stroke and/or CHD were included. Furthermore, studies with a sample size less than 500 and articles published before 2000 were excluded.

\subsection{Data Extraction}

For each study, information was extracted on author and publication year, country, sample size, gender, percentage of smokers, type of disease, method of diagnosis, dietary assessment method, disease cases, saturated fat intake, covariates adjusted, and relative risk of stroke or CHD comparing extreme quintile of saturated fat diet, and $95 \%$ CIs.

\section{Results}

Figure 1 illustrates the process of selecting the articles for this systematic review. Overall, 553 articles were obtained, 201 from ProQuest and 352 from Midline. After removing duplicates, 263 articles remained. A total of 117 articles were excluded from this review because they were published before 2000, and 128 articles did not meet the criterion of being a prospective cohort study. In addition, 4 articles were excluded because they focused only on CVD without any mention of CHD or stroke. Therefore, 16 articles were analysed in this systematic review. 


\section{3 of records included after duplicates removed}

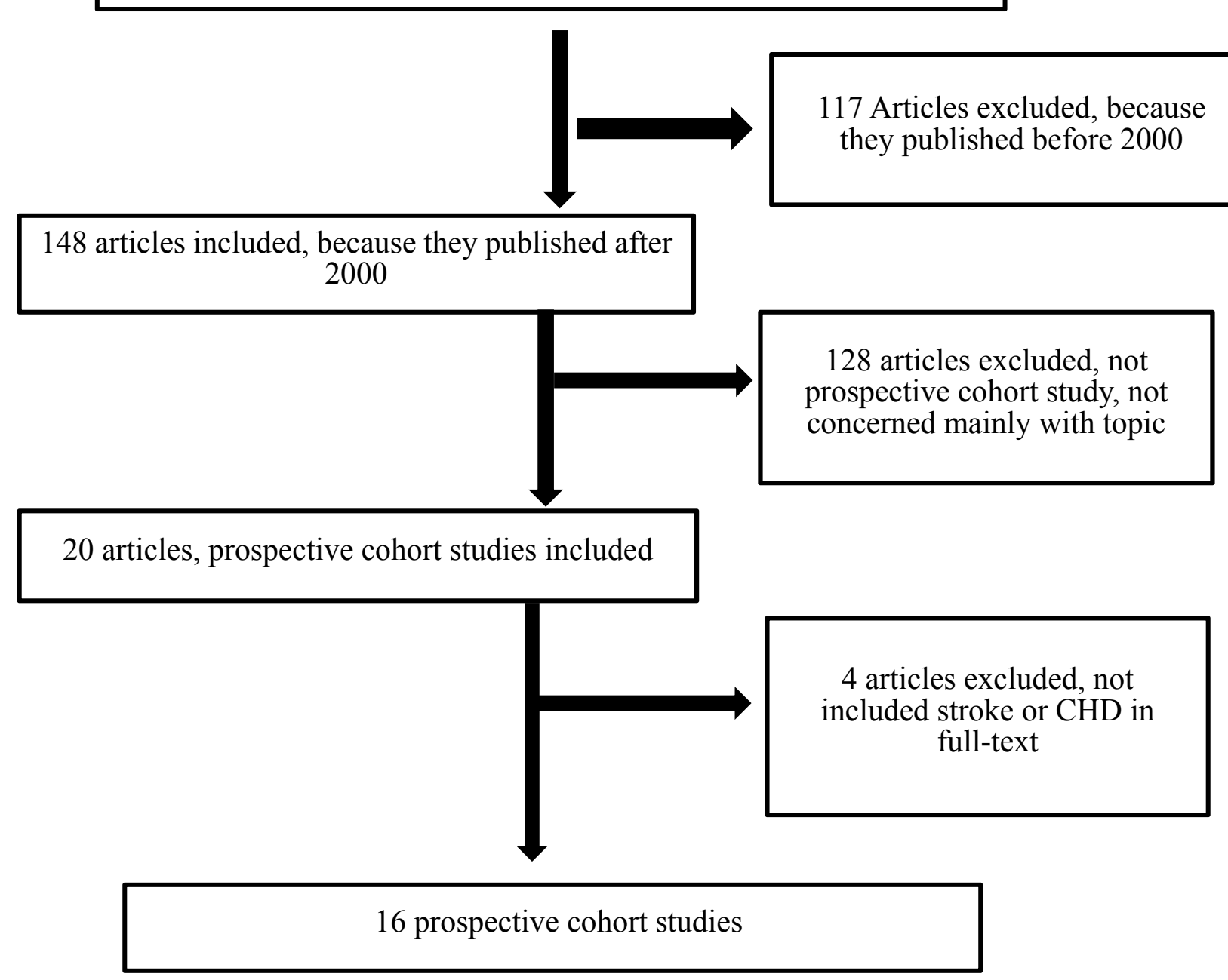

Figure 1. The process of chosen the sixteen articles which are in this review

Tables 1 and 2 show a summary of the general characteristics of the 16 prospective cohort studies included in this systematic review and the measures of association between saturated fat intake and risk of stroke and CHD in each study. Eight studies are from the United States, three are from Japan, two are from Sweden, one is from the United Kingdom, and one is from Australia. The duration of follow up across the studies ranged from 7 to 30 years. The total number of participants in the 16 prospective epidemiologic studies was 668,082, ranging from 501 to 127,536 Five studies had only female participants, two studies had only male participants, and nine studies had participants of both sexes. The ages of the participants in all 16 studies ranged from 30 to 79 years. The percentage of smokers in the 11 studies that tracked this characteristic ranged from $5.6 \%$ to $37.2 \%$, but there were five studies in which smokers were not identified.

Six of the studies used both medical record and death certification as the method of diagnosis to confirm the type of disease outcome, while six studies used only death certification and four studies used only medical record as the method of diagnosis. Furthermore, 11 studies assessed dietary intake using a food frequency questionnaire (FFQ), four studies used a 24-h recall method, and two studies used a seven-day diet record method.

Seven studies focused on measuring the association between saturated fat intake and the risk of haemorrhagic and ischemic stroke, as shown in Table 2. The total number of stroke cases in all seven of these studies was 3178 , ranging from 74 to 976 . All of these studies adjusted for total energy intake in a fully adjusted model. The saturated fat intake in the compassion group ranged in the lowest quintile from 5.2 grams per day (g/d) to $20 \mathrm{~g} / \mathrm{d}$, and in the highest quintile from $17 \mathrm{~g} / \mathrm{d}$ to $36 \mathrm{~g} / \mathrm{d}$ (H. Iso et al., 2001; Yamagishi et al., 2010). Covariates such as smoking 
status, physical activity, BMI, alcohol use, hypertension, diabetes, MFUAS, PUFAs, trans fats, and fibre intake were adjusted for in all of these studies. Five out of the seven studies measured this association in participants of both genders. The highest HR (hazard ratio) in these studies was $1.49,95 \% \mathrm{CI}(0.70-3.17)$ and the lowest HR was $0.27,95 \% \mathrm{CI}(0.27-0.85)$. One study evaluated this association only in females (HR $=2.36,95 \% \mathrm{CI}(1.10-5.09))$ and one study had only male participants ( $\mathrm{HR}=1.10,95 \% \mathrm{CI}(0.72-1.68))$.

Nine studies focused on evaluating the association between saturated fat consumption and risk of CHD, and two additional studies focused on the association of saturated fat intake and both CHD and stroke risk. The total number of CHD cases in all 9 studies was 19,655 ranging from 71 to 7667 . The lowest percentage intake of saturated fat from the total energy in these studies was $7.5 \%$ in the first quintile with $\mathrm{HR}=3.4$ and $11.3 \%$ in the fifth quintile with $\mathrm{HR}=1.1$. The highest percentage intake of saturated fat was $30 \%$ in the fifth quintile with $\mathrm{HR}=$ 3.07 and $95 \%$ CI (1.54-6.11). Furthermore, three of the 11 studies measured the stratification by both gender and age for the association between risk of CHD and saturated fat intake and their relative risk (RR) findings were RR (female, age $\geq 60$ years) $=2.34,95 \%$ CI $(1.02-5.40)$ (Boniface $\&$ Tefft, 2002), and RR (female, age $<60$ years) $=$ $2.68,95 \%$ CI (1.40-5.12) (11). Two studies measured the stratification by gender for this association (RR $($ Female $)=3.07,95 \%$ CI (1.54-6.11)) (Blekkenhorst et al., 2015) and one study measured it only by age (RR (age $\leq 60$ years $)=5.17,95 \%$ CI $(1.60-16.4))(\mathrm{Xu}$, Eilat-Adar, Loria, \& Goldbourt, 2006).

Table 1. General characteristics of 16 prospective epidemiologic studies of saturated fat intake and risk of stroke and coronary heart disease (CHD)

\begin{tabular}{|c|c|c|c|c|c|c|c|c|c|}
\hline Study & Country & $\begin{array}{l}\text { Sample } \\
\text { Size }\end{array}$ & $\begin{array}{l}\text { Age } \\
(y)\end{array}$ & Sex & $\begin{array}{l}\text { Smoker } \\
(\%)\end{array}$ & $\begin{array}{l}\text { Type of } \\
\text { disease }\end{array}$ & $\begin{array}{l}\text { Method of } \\
\text { diagnosis }\end{array}$ & $\begin{array}{l}\text { Dietary } \\
\text { assessment } \\
\text { method }\end{array}$ & $\begin{array}{l}\text { Average } \\
\text { duration of } \\
\text { follow-up } \\
\text { (y) }\end{array}$ \\
\hline \multicolumn{10}{|l|}{ Stroke } \\
\hline He et al. (2003) & USA & 43732 & $40-75$ & Male & 9.5 & Stroke & $\begin{array}{l}\text { MR and } \\
\text { DC }\end{array}$ & FFQ (1 y) & 14 \\
\hline $\begin{array}{l}\text { H. Iso et al. } \\
(2001)\end{array}$ & USA & 85764 & $34-59$ & Female & 26.2 & Haemorrhagic & $\begin{array}{l}\text { MR and } \\
\text { DC }\end{array}$ & FFQ (1 y) & 14 \\
\hline $\begin{array}{l}\text { Hiroyasu Iso et al. } \\
(2003)\end{array}$ & Japan & 4775 & $40-69$ & Both & 13.5 & Haemorrhagic & $\begin{array}{l}\text { MR and } \\
\text { DC }\end{array}$ & $\begin{array}{l}\text { 24-h recall (1 } \\
\text { d) }\end{array}$ & 14 \\
\hline $\begin{array}{l}\text { Sauvaget, } \\
\text { Nagano, Hayashi, } \\
\text { and Yamada } \\
(2004)\end{array}$ & Japan & 3731 & $35-89$ & Both & $26-35$ & Ischemic & Dc & 1-d diet record & 14 \\
\hline $\begin{array}{l}\text { Leosdottir, } \\
\text { Nilsson, Nilsson, } \\
\text { and Berglund } \\
(2007)^{2}\end{array}$ & Sweden & 28098 & $45-73$ & Both & 29.5 & Ischemic & $\mathrm{DC}$ & $\begin{array}{l}\text { FFQ }(1 \mathrm{y}) \text { and } \\
7-\mathrm{d} \\
\text { menu-diary }\end{array}$ & 8 \\
\hline $\begin{array}{l}\text { Yamagishi et al. } \\
(2010)^{2}\end{array}$ & Japan & 58453 & $40-79$ & Both & 5.6 & ischemic & $\mathrm{DC}$ & $\begin{array}{l}\text { FFQ (unknown } \\
\text { of time frame) }\end{array}$ & 14.1 \\
\hline $\begin{array}{l}\text { Wallström et al. } \\
\text { (2012) }\end{array}$ & Sweden & 20674 & $44-73$ & Both & 27 & ischemic & $\mathrm{DC}$ & $\begin{array}{l}\text { FFQ (1 y) and } \\
7-d \\
\text { menu-dietary }\end{array}$ & 13.5 \\
\hline \multicolumn{10}{|l|}{ CHD } \\
\hline $\begin{array}{l}\text { Boniface and } \\
\text { Tefft (2002) }\end{array}$ & UK & 2676 & $40-75$ & Both & NR & CHD & $\mathrm{DC}$ & $\begin{array}{l}\text { FFQ } \\
\text { ( unknown of } \\
\text { time frame) }\end{array}$ & 16 \\
\hline $\begin{array}{l}\text { Jakobsen, Schroll, } \\
\text { Heitmann, } \\
\text { Overvad, and } \\
\text { Dyerberg (2004) }\end{array}$ & Denmark & 3686 & $30-71$ & Both & NR & CHD & MR & $\begin{array}{l}\text { 7-d diet } \\
\text { records }\end{array}$ & 16 \\
\hline
\end{tabular}




\begin{tabular}{|c|c|c|c|c|c|c|c|c|c|}
\hline $\begin{array}{l}\text { Leosdottir et al. } \\
(2007)^{2}\end{array}$ & Sweden & 28098 & $45-73$ & Both & 29.5 & CHD & $\mathrm{DC}$ & $\begin{array}{l}\text { FFQ (1 y) and } \\
7-d \\
\text { menu-dietary }\end{array}$ & 8 \\
\hline $\begin{array}{l}\text { Tucker et al. } \\
\text { (2005) }\end{array}$ & USA & 501 & $37-80$ & Male & 21.8 & CHD & $\begin{array}{l}\text { MR and } \\
\text { DC }\end{array}$ & $\begin{array}{l}\text { 7-d diet } \\
\text { records }\end{array}$ & 18 \\
\hline $\begin{array}{l}\text { Xu, Eilat-Adar, } \\
\text { Loria, Goldbourt, } \\
\text { et al. (2006) }\end{array}$ & USA & 2938 & $47-79$ & Both & 29.7 & CHD & $\begin{array}{l}\text { MR and } \\
\text { DC }\end{array}$ & $\begin{array}{l}\text { 24-h recall (1 } \\
\text { d) }\end{array}$ & 7 \\
\hline $\begin{array}{l}\text { Oh, Hu, Manson, } \\
\text { Stampfer, and } \\
\text { Willett (2005) }\end{array}$ & USA & 78778 & $30-55$ & Female & NR & CHD & MR & FFQ ( 1 y) & 20 \\
\hline Li et al. (2015) & USA & 127536 & $30-75$ & Both & NR & CHD & $\begin{array}{l}\text { MR and } \\
\text { DC }\end{array}$ & FFQ (4 Y) & $24-30$ \\
\hline $\begin{array}{l}\text { Blekkenhorst et } \\
\text { al. (2015) }\end{array}$ & Australia & 1469 & $30-75$ & Female & 37.2 & CHD & $\mathrm{DC}$ & FFQ (1 y) & 10 \\
\hline $\begin{array}{l}\text { Yamagishi et al. } \\
(2010)^{2}\end{array}$ & Japan & 58453 & $40-79$ & Both & 5.6 & CHD & DC & $\begin{array}{l}\text { FFQ (unknown } \\
\text { of time frame) }\end{array}$ & 14.1 \\
\hline Zong et al. (2016) & USA & 115782 & $30-75$ & Both & 22 & CHD & $\begin{array}{l}\text { MR and } \\
\text { DC }\end{array}$ & $\begin{array}{l}\text { FFQ }(1 \mathrm{y}) \text { and } \\
7-\mathrm{d} \\
\text { menu-dietary }\end{array}$ & $24-28$ \\
\hline $\begin{array}{l}\text { Tanasescu, Cho, } \\
\text { Manson, and Hu } \\
\text { (2004) }\end{array}$ & USA & 2938 & $47-79$ & Both & 26 & CHD & MR & $\begin{array}{l}\text { 24-h diet recall } \\
(1 \mathrm{~d})\end{array}$ & 11 \\
\hline
\end{tabular}

1 NR: not reported, FFQ: food frequency questionnaire, y: year, d: day, MR: medical records, DC: death certificate.

2 Studies provided both stroke and CHD outcomes.

Table 2. Summary of outcomes for seven prospective epidemiologic studies in measuring the association of saturated fat and risk of stroke ${ }^{1}$

\begin{tabular}{|c|c|c|c|c|}
\hline Study & $\begin{array}{l}\text { Type (No. of } \\
\text { cases) }\end{array}$ & $\begin{array}{l}\text { Saturated fat } \\
\text { intake (comparing } \\
\text { group) }\end{array}$ & Adjusted covariates & Relative risk (RR) $(95 \% \mathrm{CI})$ \\
\hline He et al. (2003) & $\begin{array}{l}\text { Stroke } \quad(\text { not } \\
\text { specify) (598) }\end{array}$ & $\begin{array}{ll}-\quad \text { Fifth } & \text { quintile } \\
(36 \%) & \\
\text {-First } & \text { quintile } \\
(30 \%) & \end{array}$ & $\begin{array}{l}\text { Smoking status, physical activity, } \\
\text { BMI, alcohol consumption, fibre, } \\
\text { aspirin use potassium, other fats } \\
\text { (MFUAS, PUFAs, and trans fats), } \\
\text { fruit and vegetables. }\end{array}$ & $\begin{array}{l}-\mathrm{RR}(\text { overall })=1.10(0.72 \text { to } \\
1.68) \\
-\mathrm{RR}(\text { age }<60 \mathrm{y})=0.72 \\
(0.35-1.52) \\
-\mathrm{RR}(\text { age }>=60 \mathrm{y})=0.82(0.49- \\
1.36)\end{array}$ \\
\hline H. Iso et al. (2001) & $\begin{array}{l}\text { Haemorrhagic } \\
\text { (74) }\end{array}$ & $\begin{array}{l}\text { Fifth quintile }(40 \% \\
\mathrm{g} / \mathrm{d}) \\
\text { First quintile }(36 \% \\
\mathrm{g} / \mathrm{d})\end{array}$ & $\begin{array}{l}\text { Smoking status, age BMI, alcohol } \\
\text { consumption, menopausal status, } \\
\text { and physical activity }\end{array}$ & $-\mathrm{RR}=2.36(1.10-5.09)$ \\
\hline $\begin{array}{l}\text { Hiroyasu Iso et al. } \\
(2003)\end{array}$ & $\begin{array}{l}\text { Haemorrhagic } \\
(67)\end{array}$ & $\begin{array}{l}\text {-Fourth } \quad \text { quintile } \\
(30 \%) \\
\text {-First } \\
(20 \%)\end{array}$ & $\begin{array}{l}\text { Sex, age, total energy intake, } \\
\text { diabetes, BMI, hypertension, } \\
\text { cholesterol, smoking status, and } \\
\text { menopausal status (women) }\end{array}$ & $-\mathrm{RR}=0.30(0.13-0.71)$ \\
\hline $\begin{array}{l}\text { Sauvaget et al. } \\
(2004)\end{array}$ & Ischemic (60) & $\begin{array}{l}\text {-Third tertile }(10 \%) \\
\text {-First tertile }(4 \%)\end{array}$ & $\begin{array}{l}\text { Sex, age. Radiation dose, BMI, } \\
\text { city, smoking status, alcohol, } \\
\text { hypertension, and diabetes }\end{array}$ & $\mathrm{HR}=1.49[0.70$ to 3.17$]$ \\
\hline
\end{tabular}




\begin{tabular}{|c|c|c|c|c|}
\hline $\begin{array}{l}\text { Leosdottir et al. } \\
(2007) 2\end{array}$ & Ischemic (648) & $\begin{array}{l}\text { Male: } \\
\text { Fourth quintile } \\
(22.3 \% \text { of total } \\
\text { energy), first } \\
\text { quartile }(12.3 \%) \\
\text { Female: } \\
\text { Fourth quintile } \\
(21.8 \%), \quad \text { first } \\
\text { quartile }(12.2 \%)\end{array}$ & $\begin{array}{l}\text { Smoking status, age. Alcohol } \\
\text { consumption, marital status, } \\
\text { socioeconomic, physical activity, } \\
\text { BMI, blood pressure, and fibre } \\
\text { intake }\end{array}$ & $\begin{array}{l}-\mathrm{RR}(\text { male })=1.19(0.80-1.77) \\
-\mathrm{RR}(\text { female })=1.26(0.81-1.96)\end{array}$ \\
\hline $\begin{array}{l}\text { Yamagishi et al. } \\
(2010) 2\end{array}$ & Ischemic (976) & $\begin{array}{l}\text {-Highest } \\
(20.3 \% \text { of } r \text { total } \\
\text { energy) } \\
\text {-lowest } \\
(9.2 \% \text { of } r \text { total } \\
\text { energy) }\end{array}$ & $\begin{array}{l}\text { BMI, hypertension, diabetes, } \\
\text { smoking status, physical activity, } \\
\text { cholesterol, MUFAs, PUFAs, } \\
\text { protein, fruit and vegetables. }\end{array}$ & $\begin{array}{l}-\mathrm{RR}=0.27(0.27-0.85) \\
-\mathrm{HR} \quad(\text { total } \quad \text { stroke })=0.69 \\
(0.53-0.89)\end{array}$ \\
\hline $\begin{array}{l}\text { Wallström et al. } \\
(2012)\end{array}$ & Ischemic (755) & $\begin{array}{l}\text {-Fifth quintile }(30 \% \\
\text { of total energy) } \\
\text { - First quintile ( } 20 \% \\
\text { of total energy) }\end{array}$ & $\begin{array}{l}\text { BMI, Education level, smoking } \\
\text { status, and alcohol use }\end{array}$ & $\mathrm{HR}=0.87(0.66-1.14)$ \\
\hline $\begin{array}{l}\text { Boniface and Tefft } \\
\text { (2002) }\end{array}$ & 155 & $\begin{array}{l}\text {-Fifth quintile } \\
(8.2 \% \text { of total } \\
\text { energy) } \\
\text {-First quintile }(7.2 \% \\
\text { of total energy) }\end{array}$ & $\begin{array}{l}\text { Alcohol use, age. Smoking status, } \\
\text { and social class }\end{array}$ & $\begin{array}{l}-\mathrm{RR}(\text { male })=1.00(0.86-1.18) \\
\mathrm{RR}(\text { female })=1.40(1.09-1.79) \\
\text {-RR (male, age }<60 \mathrm{y})=1.51 \\
(0.69-3.31) \\
\text {-RR (male, age }>=60 \mathrm{y})=1.01 \\
(0.57-1.80) \\
\text {-RR (female, age }<60 \mathrm{y})=1.32 \\
(0.38-4.57) \\
\text {-RR (female, age }>=60 \mathrm{y})=2.34 \\
(1.02-5.40)\end{array}$ \\
\hline $\begin{array}{l}\text { Jakobsen et al. } \\
(2004)\end{array}$ & 326 & $\begin{array}{l}\text {-Third }(24.8 \% \text { of } \\
\text { total energy) } \\
\text {-first (14.5\% of } \\
\text { total energy) }\end{array}$ & $\begin{array}{l}\text { Protein, other fat, history of MI, } \\
\text { smoking status, education, } \\
\text { physical activity, alcohol, fibre, } \\
\text { BMI, and cholesterol }\end{array}$ & $\begin{array}{l}\text {-HR }(\text { male, Age }<60 \mathrm{y})=1.28 \\
(0.87-1.91) \\
\text {-HR }(\text { male, age }>=60 \mathrm{y})=0.94 \\
(0.70-1.28) \\
\text {-HR }(\text { female, age }<60 \mathrm{y})=2.68 \\
(1.40-5.12) \\
\text {-HR }(\text { female, age }>=60 \mathrm{y})=1.22 \\
(0.86-1.71)\end{array}$ \\
\hline $\begin{array}{l}\text { Leosdottir et al. } \\
(2007)^{2}\end{array}$ & 908 & $\begin{array}{l}\text {-Male: } \\
\text { Fourth quintile } \\
(22.3 \% \text { of total } \\
\text { energy), First } \\
\text { quintile }(12.3 \% \text { of } \\
\text { total energy) } \\
\text {-Female: } \\
\text { Fourth quintile } \\
(21.8 \% \text { of total } \\
\text { energy), First } \\
\text { quintile (12.2\% of } \\
\text { total energy) }\end{array}$ & $\begin{array}{l}\text { Smoking status, alcohol use, age, } \\
\text { physical activity, BMI, fibre, blood } \\
\text { pressure, and marital status }\end{array}$ & $\begin{array}{l}-\mathrm{HR}(\text { male })=1.05(0.83-1.34) \\
-\mathrm{HR}(\text { female })=0.98(0.71-1.33)\end{array}$ \\
\hline
\end{tabular}




\begin{tabular}{|c|c|c|c|c|}
\hline \multirow[b]{2}{*}{ Tucker et al. (2005) } & \multirow[b]{2}{*}{71} & -Third tertile & \multirow[b]{2}{*}{$\begin{array}{l}\text { BMI, age, smoking status, } \\
\text { physical activity, alcohol use, } \\
\text { supplement use, fruit and } \\
\text { vegetable intake }\end{array}$} & \multirow{2}{*}{$\begin{array}{l}-R R(\text { overall })=1.04(0.99-1.08) \\
-R R \quad(\text { age }<60 \text { y) }=0.57 \\
(0.14-2.30) \\
-R R(\text { age }>=60 \\
(0.73-7.27)\end{array}$} \\
\hline & & $\begin{array}{l}(14.0 \% \text { of total } \\
\text { energy) } \\
\text { - First tertile }(12.3 \% \\
\text { of total energy) }\end{array}$ & & \\
\hline $\begin{array}{l}\text { Xu, Eilat-Adar, } \\
\text { Loria, Goldbourt, } \\
\text { et al. (2006) }\end{array}$ & 138 & $\begin{array}{l}\text {-Fourth quintile } \\
(16.5 \% \text { of total } \\
\text { energy) } \\
\text {-First quintile }(7.5 \% \\
\text { of total energy) }\end{array}$ & $\begin{array}{l}\text { Sex, age, diabetes, BMI, HDL, } \\
\text { LDL, TG, smoking, alcohol } \\
\text { consumption, hypertension, and } \\
\text { energy from protein }\end{array}$ & $\begin{array}{l}-\mathrm{RR} \quad(\text { age }<60 \text { y) }=5.17 \\
(1.60-16.4) \\
-\mathrm{RR}(\text { age }>=60 \\
(0.41-1.54) \\
-\mathrm{HR}=3.4(1.17,10.04)\end{array}$ \\
\hline Oh et al. (2005) & 1766 & $\begin{array}{l}\text {-Fifth quintile } \\
(17.6 \% \text { of total } \\
\text { energy) } \\
\text {-First } \\
(10.1 \% \text { of total } \\
\text { energy) }\end{array}$ & $\begin{array}{l}\text { BMI, age, smoking status, alcohol } \\
\text { use, hypertension, menopausal } \\
\text { status, aspirin use, supplement use, } \\
\text { protein, cholesterol, MUFAS, } \\
\text { PUFAs, trans fat, and fruit and } \\
\text { vegetables }\end{array}$ & $\mathrm{RR}=1.52(1.30-1.79)$ \\
\hline Li et al. (2015) & 7,667 & $\begin{array}{l}\text {-Fifth quintile } \\
(16.9 \% \text { of total } \\
\text { energy) } \\
\text {-First quintile }(9.6 \% \\
\text { of total energy) }\end{array}$ & $\begin{array}{l}\text { Age, white people, alcohol } \\
\text { consumption, smoking status, } \\
\text { supplement use, hypertension, } \\
\text { physical activity, aspirin use, fruit } \\
\text { and vegetables }\end{array}$ & $-\mathrm{HR}=1.54(1.38-1.73)$ \\
\hline $\begin{array}{l}\text { Blekkenhorst et al. } \\
\text { (2015) }\end{array}$ & 134 & $\begin{array}{ll}\text {-highest } & \text { quintile } \\
(30 \%) & \\
\text {-lowest } & \text { quintile } \\
(17 \% \mathrm{~g} / \mathrm{d}) & \end{array}$ & $\begin{array}{l}\text { Age, cholesterol, physical activity, } \\
\text { smoking status, diabetes, aspirin } \\
\text { use, protein, fibre, MUFAs, and } \\
\text { PUFAs }\end{array}$ & $-\mathrm{HR}=3.07(1.54-6.11)$ \\
\hline $\begin{array}{l}\text { Yamagishi et al. } \\
(2010)^{2}\end{array}$ & 836 & $\begin{array}{l}\text {-Highest quintile } \\
(20.3 \% \text { of total } \\
\text { energy) } \\
\text {-lowest quintile } \\
(9.2 \% \text { of total } \\
\text { energy) }\end{array}$ & $\begin{array}{l}\text { BMI, hypertension, diabetes, } \\
\text { smoking status, physical activity, } \\
\text { cholesterol, MUFAs, PUFAs, } \\
\text { protein, fruit and vegetables. }\end{array}$ & $-\mathrm{HR}=1.54(1.38-1.73)$ \\
\hline Zong et al. (2016) & 7035 & $\begin{array}{l}\text {-Fifth quintile } \\
(11.3 \% \text { of total } \\
\text { energy) } \\
\text {-First quintile }(8.2 \% \\
\text { of total energy) }\end{array}$ & $\begin{array}{l}\text { Age, alcohol use, smoking status, } \\
\text { supplement use, hypertension, } \\
\text { physical activity, BMI, fruit and } \\
\text { vegetables }\end{array}$ & $-\mathrm{HR}=1.18(1.09-1.28)$ \\
\hline $\begin{array}{l}\text { Tanasescu et al. } \\
(2004)\end{array}$ & 619 & $\begin{array}{l}\text {--Fifth quintile } \\
(19.1 \% \text { of total } \\
\text { energy) } \\
\text {-First quintile } \\
(10.8 \% \text { of total } \\
\text { energy) }\end{array}$ & $\begin{array}{l}\text { BMI, smoking status, cholesterol, } \\
\text { blood pressure, diabetes, alcohol } \\
\text { use, supplement use, and physical } \\
\text { activity. }\end{array}$ & $-\mathrm{RR}=1.37(1.12-1.68)$ \\
\hline
\end{tabular}

\footnotetext{
${ }^{1}$ MUFA: monounsaturated fatty acid, PUFA: polyunsaturated fatty acid, HR: hazard ratio, NR: not reported, and BMI: body mass index.
}

\section{Discussion}

As previously stated, this systematic review sought to evaluate the effects of saturated fat intake on risk of CVD by 
summarising the data from 16 prospective cohort studies. The World Health Organization (2017) has pointed out that in $2015,38 \%$ of CVD-related deaths were due to stroke and $42 \%$ to CHD. Consequently, this review focuses on the association of saturated fat consumption with only these two types of CVD, and it has found that there is an inverse association between a diet high in saturated fat diet and stroke and a positive association with CHD after adjusting for other nutrients and non-nutrients (such as fibre, physical activity, alcohol use, smoking status, MUFAs (monounsaturated fatty acids), PUFAs (polyunsaturated fatty acids) and trans fats) wherever possible. In addition, the subgroup analysis shows that sex, age, sample size and smoking status can all affect the association of saturated fat intake with stroke and CHD.

All 16 studies relied on the accuracy of dietary assessments using various methods, all of which were dependent on self-reporting. Raymond J. Carroll et al. (2012) pointed out that 4- to 7-day food records are the most accurate means for dietary assessment; however, this method is not feasible in large cohort studies. On the other hand, while a single 24-hour recall is relatively easy to collect, this information is not sufficient for large cohort epidemiologic studies. Food frequency questionnaires (FFQs) have become the preferred method in large epidemiologic studies because they can inexpensively evaluate long-term diet patterns (Raymond J Carroll et al., 2012), although they are subject to systematic and random errors. Eleven of the 16 studies summarised here used the FFQ method to assess dietary patterns, which should be considered a strength of this review.

This review found an inverse, insignificant association between saturated fat intake and risk of stroke (He et al., 2003; Hiroyasu Iso et al., 2003; Yamagishi et al., 2010). This could be related to the controlling of other nutrients in the diet, such as polyunsaturated fat, to replace the energy lost from not eating saturated fat. Recent epidemiological studies support the finding that replacing saturated fat with polyunsaturated fat is more beneficial for decreasing stroke and CVD risk than exchanging monounsaturated fat or carbohydrates for saturated fat in the diet (Micha \& Mozaffarian, 2010). Therefore, the risk of stroke and CVD could be increased with a high carbohydrate diet even with increasing saturated fat or polyunsaturated fat. On the other hand, H. Iso et al. (2001) found that the risk of haemorrhage had a strong association with saturated fat intake. It could be that some types of stroke are associated with saturated fat intake, but in this review, there was insufficient evidence from the previous studies about this association. Gray (2010) pointed out that there are differences in the association between saturated fat intake and mortality for each of three main types of stroke: haemorrhagic, ischemic and transient ischemic attacks.

Most of studies in this review showed a weak (Li et al., 2015; Oh et al., 2005; Yamagishi et al., 2010; Zong et al., 2016) to strong (Blekkenhorst et al., 2015) significant association of saturate fat intake with CHD risk. Therefore, a diet high in saturated fat is a risk factor for CHD, whereas increasing one's intake of this type of fat is a protective factor for stroke (as mentioned above). Many epidemiologic studies support this finding of the association between saturated fat intake and an increased risk of CHD (Siri-Tarino, Sun, Hu, \& Krauss, 2010).

Several of the studies in this review showed that gender and age may modify the association of saturated fat intake with stroke and CHD risk. The male gender had an effect on the association between saturated fat and risk of stroke and CHD (He et al., 2003; Leosdottir et al., 2007), whereas the female gender was a risk factor for CHD (Blekkenhorst et al., 2015; Oh et al., 2005). Another finding in this review is the effect of gender on the association of saturated fat intake with CHD and stroke risk varies by age groups. Bots, Peters (29) has pointed out that differences in CHD and stroke mortality rates between men and women can also vary by age. In one study, age had no effect on the association of saturated fat intake with stroke and CHD in men (Tucker et al., 2005), while another study showed that for women, being under the age of 60 was a risk factor for CHD. Yet another study showed that being a female and elderly was a risk factor for CHD. (Xu, Eilat-Adar, Loria, \& Goldbourt, 2006). Therefore, there is not sufficient clear evidence from the 16 prospective cohort studies to conclude that age is a risk factor for CHD. 


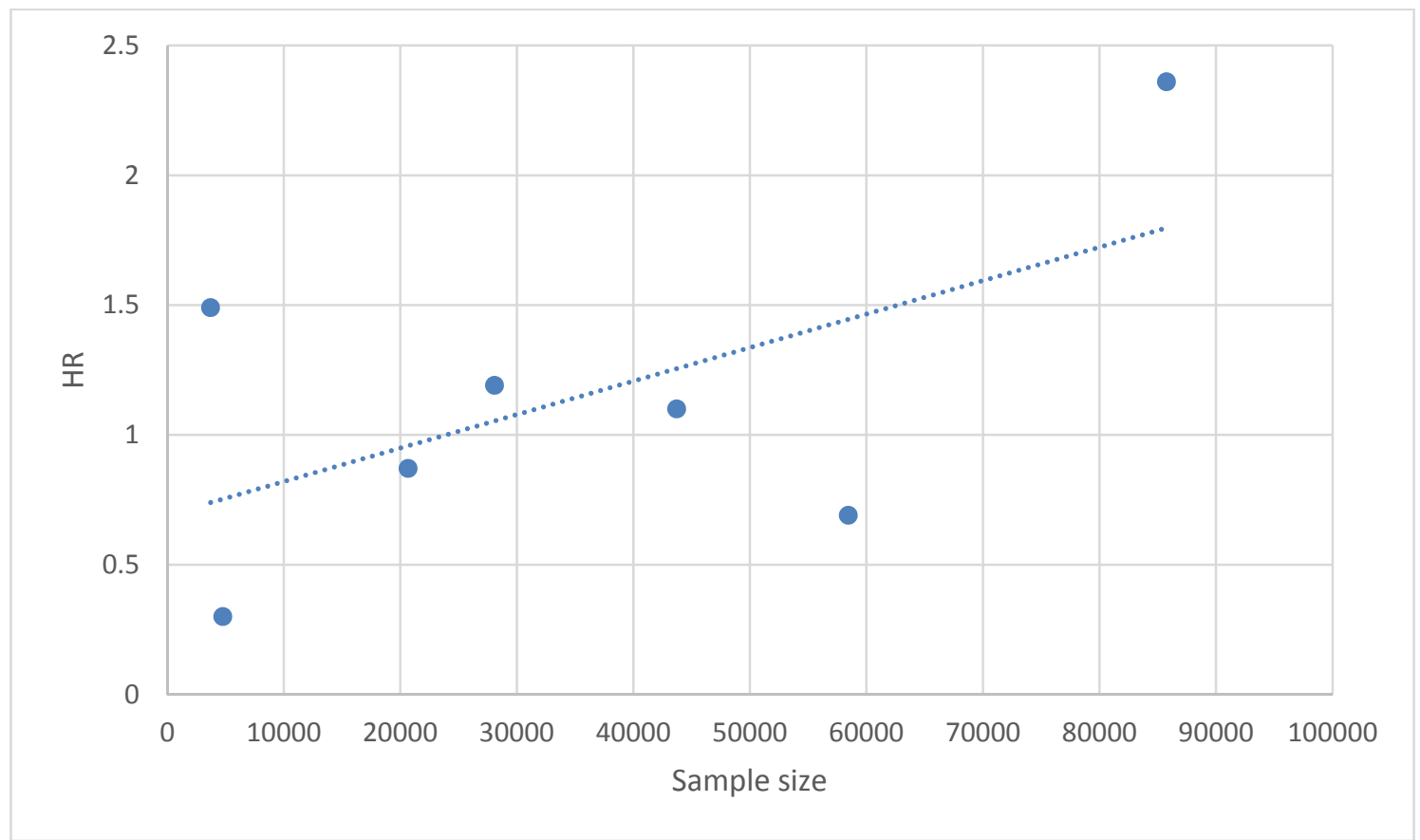

Figure 2. The relationship between the percentage of participants with a smoking history and the hazard ratio (association of saturated fat intake with stroke risk)

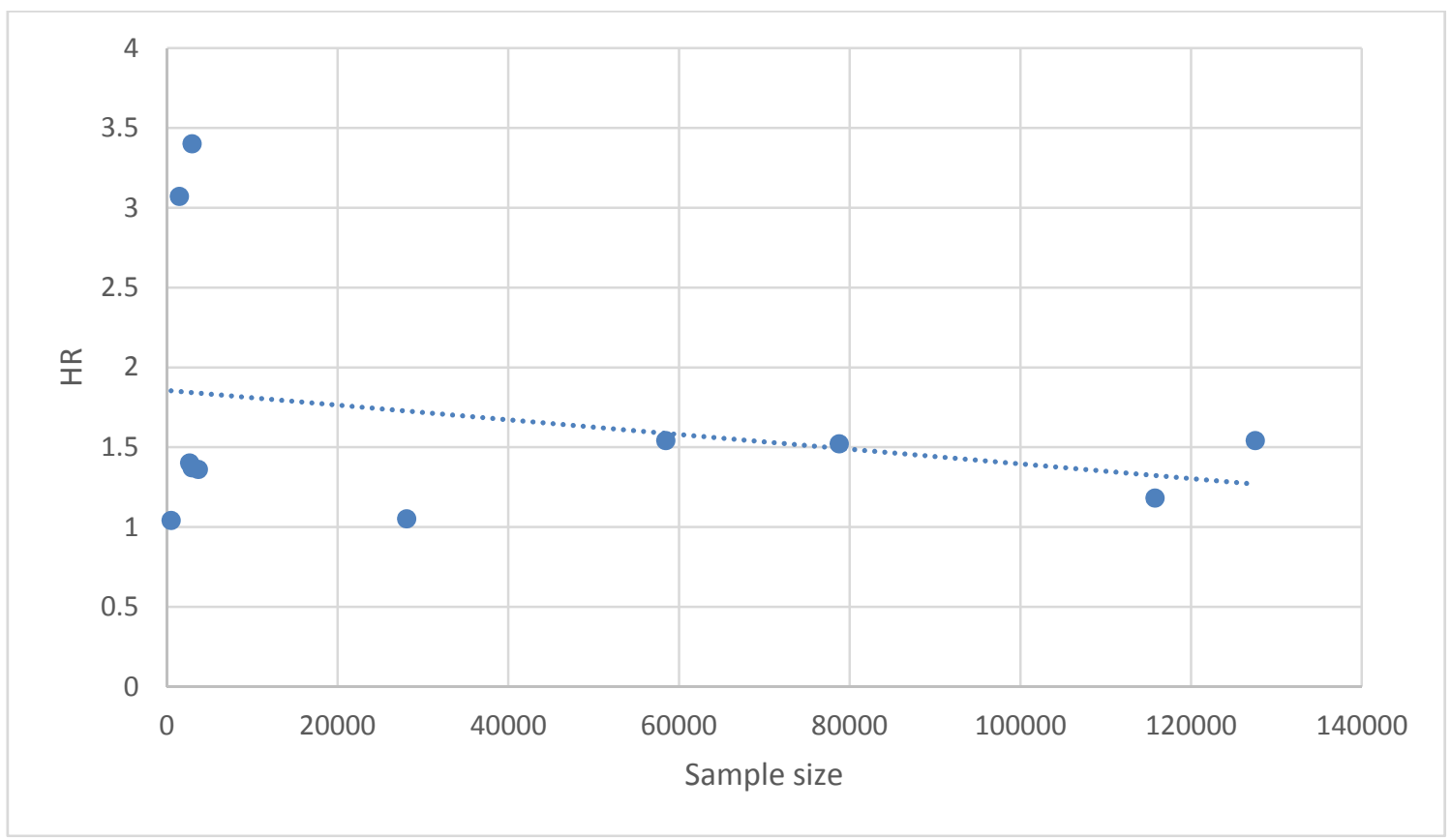

Figure 3. The relationship between percentage of participants with a smoking history and the hazard ratio (association of saturated fat intake with CHD risk)

The relationships between the sample sizes and HRs for the 16 studies included in this review for CHD and stroke are shown in Figures 2 and 3. Overall, as the sample size increases, the HR of stroke increases, whereas the HR of CHD decreases when the sample size increases. This could be caused by variations between the sample sizes in the different studies; i.e., the differences between the largest and smallest sample sizes were 82,033 participants for the stroke studies and 127,035 participants for the CHD studies. In addition, the long follow-up periods in these studies could have affected the outcomes; for example, during the follow-up period, the researchers could have controlled for other factors, such as smoking status (mentioned previously), which could reduce the bias of study 
and the risk of developing the disease (Song \& Chung, 2010). The longest follow-up period in the stroke studies was 14 years, while the longest in the CHD studies was 30 years. These difference in follow-up periods could be an explanation for the varying associations between saturated fat intake and stroke and CHD risk.

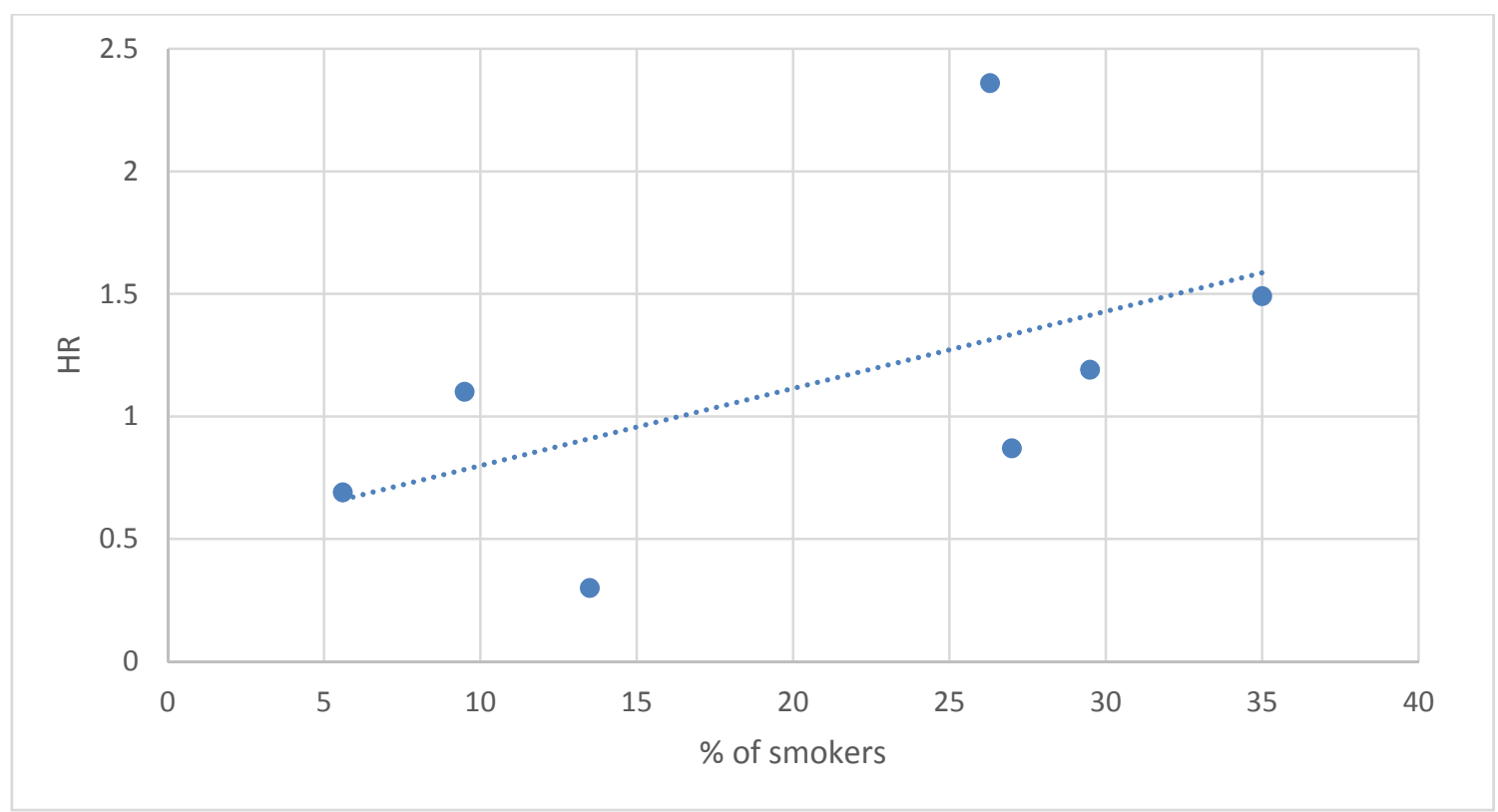

Figure 4. The relationship between the percentage of participants with a history of smoking and the hazard ratio (association of saturated fat intake with stroke risk)

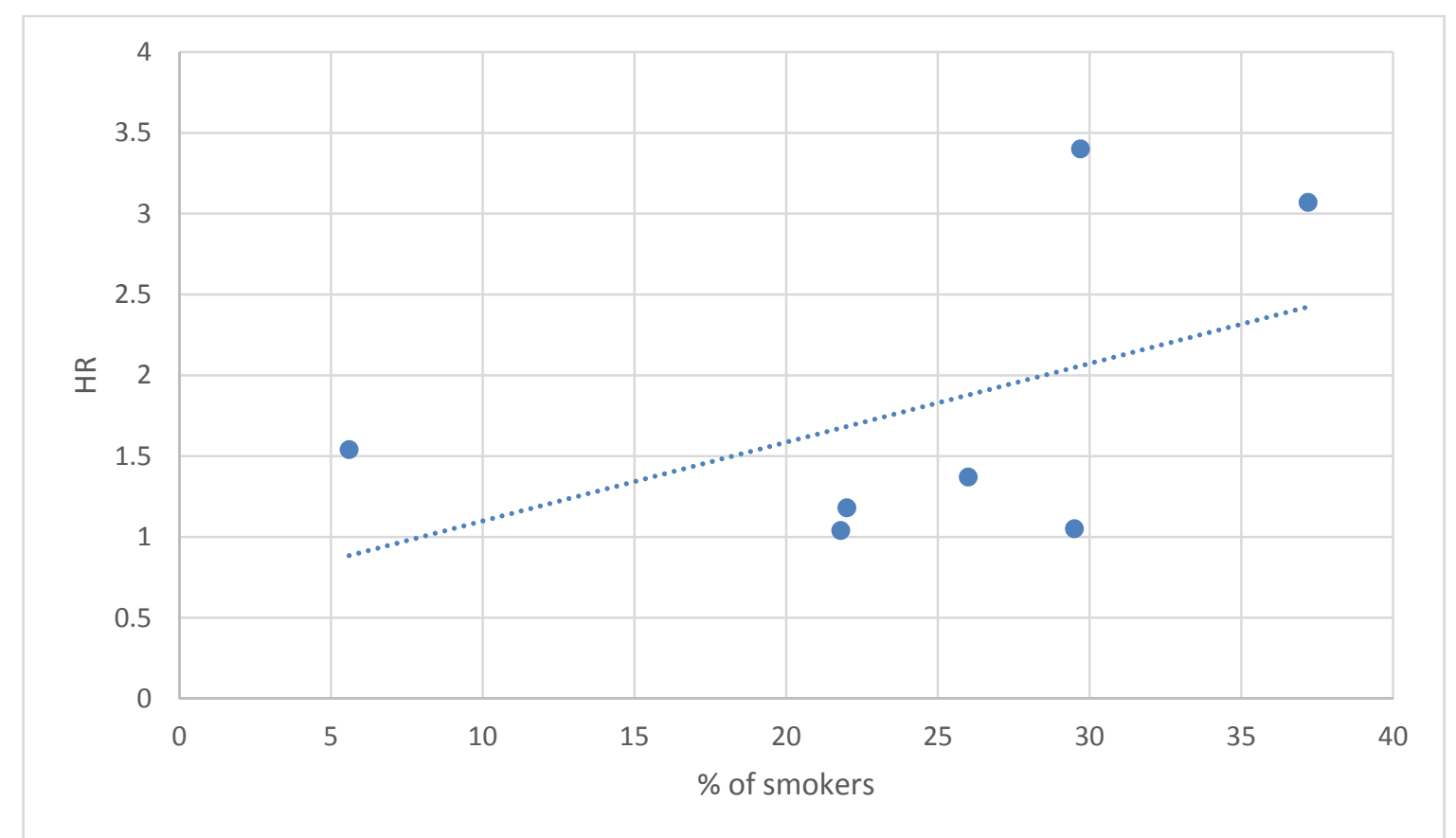

Figure 5. The relationship between the percentage of participants with a history of smoking and the hazard ratio (association of saturated fat intake with CHD risk)

Figures 4 and 5 show that the percentage of participants who had a history of smoking could have affected this association, because as the percentage of smokers increased, the HR also increased in both the stroke and CHD studies. For example, when $5.6 \%$ of the participants had a history of smoking, the HR was 0.69 , while when $37.2 \%$ 
had a history of smoking, the HR was 3.07 (see Figure 5). Therefore, for the studies that had a lower percentage of participants with a history of smoking at intake, the association of saturated fat intake with the risk of both diseases was lower. Ambrose and Barua (2004) highlighted that smokers are up to four times more likely to die from stroke and CHD than non-smokers. In addition, recent studies have reported that cigarette smoking is one of the most important risk factors for CVD (Mendelsohn, 2013 ). Therefore, in cohort studies that span short periods of time, a history of smoking can affect the association between saturated fat intake and the risk of strike and CHD, even after adjusting for this factor.

\subsection{Strength and Limitations of This Review}

This systematic review has summarised prospective cohort studies to determine risk factors for stroke and CHD; these types of studies can minimise recall error because the collections of outcomes take place at regular time intervals. In addition, this paper has included studies with large sample sizes and long periods of follow-up (ranging from 7 to 30 years). However, this review has not attempted to look at all studies focused on the association of saturated fat intake with CVD. Rather, it includes prospective cohort studies which met specific criteria for inclusion. All 16 studies included in this review used self-reported measures to assess the effect of a diet with reduced saturated fat. Eleven of the studies used FFQs as their dietary assessment methods; FFQs are considered to be a more accurate method for collecting dietary data in long-term epidemiologic studies than 24-hour recall and 1- to 7-day diet records. Some of the studies included in this review did not report subgroup analyses of age and gender and therefore overlooked their potential effects on the association between saturated fat intake and CVD.

\section{Conclusion}

This systematic review has showed that there is sufficient evidence from prospective cohort studies to conclude that saturated fat intake is associated with decreased or increased stroke, CHD and CVD risk. Moreover, the available data show that there are associations between stroke, CHD and CVD with saturated fat intake in specific gender and age subgroups. Future studies are needed that utilise proper dietary assessment methods to provide more accurate findings over long periods of time. Furthermore, more studies are needed to evaluate the association of saturated fat with CVD risk and to clarify whether both stroke risk and CHD risk are likely to be influenced by replacing saturated fat with other nutrients.

\section{Competing Interests Statement}

The authors declare that there are no competing or potential conflicts of interest.

\section{References}

Ambrose, J. A., \& Barua, R. S. (2004). The pathophysiology of cigarette smoking and cardiovascular disease: an update. Journal of the American college of cardiology, 43(10), 1731-1737. https://doi.org/10.1016/j.jacc.2003.12.047

Australian Institute of Health and Welfare [AIHW]. (2011). Cardiovascular disease: Australian facts 2011. Retrieved from http://www.aihw.gov.au/publication-detail/?id=10737418510\&tab=2

Blekkenhorst, L. C., Prince, R. L., Hodgson, J. M., Lim, W. H., Zhu, K., Devine, A., . . L Lewis, J. R. (2015). Dietary saturated fat intake and atherosclerotic vascular disease mortality in elderly women: A prospective cohort study. The American journal of clinical nutrition, 101(6), 1263. https://doi.org/10.3945/ajcn.114.102392

Boniface, D. R., \& Tefft, M. E. (2002). Dietary fats and 16-year coronary heart disease mortality in a cohort of men and women in Great Britain. European Journal of Clinical Nutrition, 56(8), 786. https://doi.org/10.1038/sj.ejcn.1601509

Carroll, R. J., Midthune, D., Subar, A. F., Shumakovich, M., Freedman, L. S., Thompson, F. E., \& Kipnis, V. (2012). Taking Advantage of the Strengths of 2 Different Dietary Assessment Instruments to Improve Intake Estimates for Nutritional Epidemiology. American Journal of Epidemiology, 175(4), 340-347. https://doi.org/10.1093/aje/kwr317

Carroll, R. J., Midthune, D., Subar, A. F., Shumakovich, M., Freedman, L. S., Thompson, F. E., \& Kipnis, V. (2012). Taking advantage of the strengths of 2 different dietary assessment instruments to improve intake estimates for nutritional epidemiology. American journal of epidemiology, kwr317. https://doi.org/10.1093/aje/kwr317

Gray, N. (2010). Low saturated fat intake associated with higher stroke mortality. Retrieved from 
http://www.foodnavigator.com/Science/Low-saturated-fat-intake-associated-with-higher-stroke-mortality

He, K., Merchant, A., Rimm, E. B., Rosner, B. A., Stampfer, M. J., Willett, W. C., \& Ascherio, A. (2003). Dietary Fat Intake And Risk Of Stroke In Male Us Healthcare Professionals: 14 Year Prospective Cohort Study. BMJ: British Medical Journal, 327(7418), 777-781. https://doi.org/10.1136/bmj.327.7418.777

Iso, H., Sato, S., Kitamura, A., Naito, Y., Shimamoto, T., \& Komachi, Y. (2003). Fat and Protein Intakes and Risk of Intraparenchymal Hemorrhage among Middle-aged Japanese. American Journal of Epidemiology, 157(1), 32-39. https://doi.org/10.1093/aje/kwf166

Iso, H., Stampfer, M. J., Manson, J. E., Rexrode, K., Hu, F., Hennekens, C. H., . . W Willett, W. C. (2001). Prospective study of fat and protein intake and risk of intraparenchymal hemorrhage in women. Circulation, 103(6), 856. https://doi.org/10.1161/01.CIR.103.6.856

Jakobsen, M. U., Schroll, M., Heitmann, B. L., Overvad, K., \& Dyerberg, J. (2004). Dietary fat and risk of coronary heart disease: Possible effect modification by gender and age. American Journal of Epidemiology, 160(2), 141-149. https://doi.org/10.1093/aje/kwh193

Leosdottir, M., Nilsson, P. M., Nilsson, J.-Å., \& Berglund, G. (2007). Cardiovascular event risk in relation to dietary fat intake in middle-aged individuals: data from The Malmö Diet and Cancer Study. European Journal of Cardiovascular Prevention \& Rehabilitation, 14(5), 701-706. http://dx.doi.org/10.1097/01.HJR.0b013e3282a56c45

Li, Y., Hruby, A., Bernstein, A. M., Ley, S. H., Wang, D. D., Chiuve, S. E., . . Hu, F. B. (2015). Saturated Fats Compared With Unsaturated Fats and Sources of Carbohydrates in Relation to Risk of Coronary Heart Disease: A Prospective Cohort Study: A Prospective Cohort Study. Journal of the American College of Cardiology, 66(14), 1538-1548. https://doi.org/10.1016/j.jacc.2015.07.055

Mendelsohn, C. (2013). Smoking and cardiovascular disease. Retrieved from http://colinmendelsohn.com.au/files/8613/8473/4175/Mendelsohn_CP._Smoking_and_Cardiovascular_Dise ase._Cardiology_Today_20133423-25.pdf

Micha, R., \& Mozaffarian, D. (2010). Saturated fat and cardiometabolic risk factors, coronary heart disease, stroke, and diabetes: A fresh look at the evidence. Lipids, 45(10), 893-905. https://doi.org/10.1007/s11745-010-3393-4

Oh, K., Hu, F. B., Manson, J. E., Stampfer, M. J., \& Willett, W. C. (2005). Dietary fat intake and risk of coronary heart disease in women: 20 years of follow-up of the nurses' health study. American Journal of Epidemiology, 161(7), 672-679. https://doi.org/10.1093/aje/kwi085

Ram, R. V., \& Trivedi, A. V. (2012). Behavioral risk factors of coronary artery disease: A paired matched case control study. Journal of cardiovascular disease research, 3(3), 212-217. https://doi.org/10.4103/0975-3583.98896

Sauvaget, C., Nagano, J., Hayashi, M., \& Yamada, M. (2004). Animal protein, animal fat, and cholesterol intakes and risk of cerebral infarction mortality in the adult health study. Stroke, 35(7), 1531-1537. https://doi.org/10.1161/01.STR.0000130426.52064.09

Siri-Tarino, P. W., Sun, Q., Hu, F. B., \& Krauss, R. M. (2010). Saturated fatty acids and risk of coronary heart disease: modulation by replacement nutrients. Current atherosclerosis reports, 12(6), 384-390. https://doi.org/10.1007/s11883-010-0131-6

Song, J. W., \& Chung, K. C. (2010). Observational studies: cohort and case-control studies. Plastic and reconstructive surgery, 126(6), 2234. https://doi.org/10.1097/PRS.0b013e3181f44abc

Tanasescu, M., Cho, E., Manson, J., \& Hu, F. (2004). Dietary fat and cholesterol and the risk of cardiovascular disease among women with type 2 diabetes. American Journal of Clinical Nutrition, 79(6), 999-1005. https://doi.org/10.1093/ajcn/79.6.999

The Department of Health. (2016). Cardiovascular disease. Retrieved from http://www.health.gov.au/internet/main/publishing.nsf/content/chronic-cardio

Tucker, K. L., Qiao, N., Hallfrisch, J., Muller, D., Andres, R., \& Fleg, J. L. (2005). The combination of high fruit and vegetable and low saturated fat intake is more protective against mortality in aging men than is either alone: The Baltimore Longitudinal Study of Aging. Journal of Nutrition, 135(3), 556-561. https://doi.org/10.1093/jn/135.3.556 
Wallström, P., Sonestedt, E., Hlebowicz, J., Ericson, U., Drake, I., Persson, M., . . Obukhov, A. G. (2012). Dietary Fiber and Saturated Fat Intake Associations with Cardiovascular Disease Differ by Sex in the Malmö Diet and Cancer Cohort: A Prospective Study. PLoS ONE, 7(2). https://doi.org/10.1371/journal.pone.0031637

World Health Organization. (2017). Cardiovascular diseases (CVDs). Retrieved from http://www.who.int/mediacentre/factsheets/fs317/en/

World Health Organization [WHO]. (2004). The global burden of disease. Retrieved from http://www.who.int/healthinfo/global_burden_disease/GBD_report_2004update_full.pdf

World Health Organization [WHO]. (2009). World Health statistics 2009. Retrieved from http://www.who.int/gho/publications/world_health_statistics/EN_WHS09_Full.pdf

World Health Organization [WHO]. (2017). Cardiovascular diseases (CVDs). Retrieved from http://www.who.int/mediacentre/factsheets/fs317/en/

Xu, J., Eilat-Adar, S., Loria, C., \& Goldbourt, U. (2006). Dietary fat intake and risk of coronary heart disease: the Strong Heart Study. The American Journal of Clinical Nutrition, 84(4), 894-902. https://doi.org/10.1093/ajen/84.4.894

Xu, J., Eilat-Adar, S., Loria, C., Goldbourt, U., Howard, B. V., Fabsitz, R. R., . . Lee, E. T. (2006). Dietary fat intake and risk of coronary heart disease: the Strong Heart Study. The American journal of clinical nutrition, 84(4), 894. https://doi.org/10.1093/ajcn/84.4.894

Yamagishi, K., Iso, H., Yatsuya, H., Tanabe, N., Date, C., Kikuchi, S., . . Tamakoshi, A. (2010). Dietary intake of saturated fatty acids and mortality from cardiovascular disease in Japanese: the Japan Collaborative Cohort Study for Evaluation of Cancer Risk (JACC) Study. The American journal of clinical nutrition, 92(4), 759. https://doi.org/10.3945/ajen.2009.29146

Zong, G., Li, Y., Wanders, A. J., Alssema, M., Zock, P. L., Willett, W. C., . . Sun, Q. (2016). Intake of individual saturated fatty acids and risk of coronary heart disease in US men and women: two prospective longitudinal cohort studies. BMJ, 355. https://doi.org/10.1136/bmj.i5796

\section{Copyrights}

Copyright for this article is retained by the author(s), with first publication rights granted to the journal.

This is an open-access article distributed under the terms and conditions of the Creative Commons Attribution license (http://creativecommons.org/licenses/by/4.0/). 